\section{FROM PROVINCIAL PILOTS TO NATIONAL DRINK DRIVING ENFORCEMENT POLICY IN VIET NAM}

${ }^{1}$ Nam Nguyen Phuong, ${ }^{2}$ Jon Passmore, ${ }^{3}$ Quang La Ngoc, ${ }^{4}$ Oanh Trinh To. ${ }^{1}$ WHO, Vietnam ${ }^{2}$ WHO Western Pacific Region, Manila; ${ }^{3}$ Hanoi School of Public Health, Vietnam; ${ }^{4}$ Global Road Safety Partnership, Vietnam

10.1136/injuryprev-2016-042156.629

Background Drink driving is a major risk factor for road crashes in Viet Nam. Studies have shown up to $36 \%$ motorcycle riders and $66.8 \%$ car drivers admitted to hospital had a BAC above the limit.

Activities This program partnered with National Traffic Safety Committee (NTSC), Global Road Safety Partnership (GRSP) implemented pilot enhanced enforcement of drink driving in 5 provinces of Viet Nam. Senior traffic police were trained overseas on this model followed by extensive trainings for police officers in program provinces. Police operations were supplemented by international standard breathalysers, and an intensive social marketing campaign on national and provincial media. Implementation results in different time periods were periodically briefed to senior leaders of NTSC, and General Department of Police for decision making. Independent monitoring was provided by Hanoi School of Public Health (HSPH).

Results The enforcement campaign was implemented from 2010 to December 2013. More than 200,000 road users were breath tested during high alcohol times $(12 \mathrm{pm}-2 \mathrm{pm}$ and $6 \mathrm{pm}-9$ $\mathrm{pm})$. The efficiency of enforcement improved dramatically. The number of tests given in 2013 in Quang Ninh was 22 times higher than in Bac Ninh where the new practice was not implemented.

Enforcement results showed a declining trend of violations from over $30 \%$ to less than $10 \%$ in intervention provinces. Public's awareness on drink driving has been maintained at over 90\%. Monitoring results from HSPH showed a declining mortality trend between 2010 and 2013.

Having shown high efficiency and high public acceptance of the new enforcement model, in June 2014, the General Department of Police issued a decision to implement the new enforcement practice nation-wide. In 45 days of implementation of the national enforcement campaign applying the new practice between mid-December 2014 and February 2015, traffic police penalised 35,370 drivers for violations of drink driving regulation compared with virtually none before the program.

Conclusions It's essential to design and implement solid pilot program so good quality data could be produced and presented to senior Government leaders to advocate for policy change. It's also crucial to link pilot interventions to national priorities to ensure large program impact and sustainability - an important factor for a problem that requires long term interventions.

\section{TRENDS IN PREVALENCE OF DRINK DRIVING AND SPEEDING IN TWO CHINESE TWO CITIES FROM 2010 TO 2014: EVALUATING A FIVE-YEAR PROJECT}

${ }^{1}$ Qingfeng Li, ${ }^{1}$ Huan He, ${ }^{2}$ Leilei Duan, ${ }^{2}$ Yuan Wang, ${ }^{1}$ David M Bishai, ${ }^{1}$ Adnan A Hyder. ${ }^{1} J o h n s$ Hopkins International Injury Research Unit, Department of International Health, Johns Hopkins Bloomberg School of Public Health; 'Injury Prevention Division, National Centre for Chronic and Non-Communicable Disease Control and Prevention (NCNCD), Chinese CDC, Beijing, China

10.1136/injuryprev-2016-042156.630
Background Road traffic injuries are a serious public health challenge and impose huge health and economic burden in China. To address the problem, Bloomberg Philanthropies funded a consortium of international partners to design and implement targeted interventions, such as social media campaigns, advocacy for legislative change, and law enforcement training, to reduce the percentage of drink driving and speeding in two Chinese cities from 2010 to 2014. This is one of few road safety projects in China that involve multiple international partners and domestic stakeholders.

Methods To monitor and evaluate the project, observational data on speeding were regularly collected from representative samples in both Chinese cities. Data on drinking and driving were collected in collaboration with local traffic police using breathalysers.

Results The analysis based on 15 rounds of data show that from May 2011 to Nov 2014, the percentage of vehicles driving above speed limit decreased from 31.77\% (95\% CI: 29.16-34.47) to $7.40 \%$ (95\% CI: 6.96-7.85) in Dalian and from 13.50\% (95\% CI: $11.69-15.47)$ to $6.86 \%$ (95\% CI: 6.39-7.36) in Suzhou. Drink driving decreased from 1.64\% (95\% CI: 1.10-2.36) in Jan 2011 to $0.50 \%$ (95\% CI: $0.24-0.92$ ) in Nov 2014 in Dalian and from $5.66 \%$ (95\% CI: $4.75-6.69$ ) to $0.33 \%$ (95\% CI: 0.04 $1.20)$ in Suzhou during approximately the same period. All changes are statistically significant..

Conclusions Despite the difficulty in attributing the changes to the program due to the lack of control cities, the substantial reduction in the prevalence of the two risk factors suggests that through coordinated actions, internationally recognised best practices in road safety are effective in improving Chinese road traffic safety.

\section{IMPLICATIONS OF ALCOHOL SCREENING IN PATIENTS WITH HEAD TRAUMA OF DIFFERENT MECHANISMS ON INJURY}

${ }^{1,2}$ Ayman El-Menyar, ${ }^{1}$ Hassan Al-Thani, 'Rafael Consunji. 'Trauma Surgery, Hamad General Hospital, Doha, Qatar, ${ }^{2}$ Clinical Medicine, Weill Cornell, Doha, Qatar

\subsection{6/injuryprev-2016-042156.631}

Background the effect of alcohol intoxication on the outcomes of traumatic brain injury (TBI) is debatable although recent data are in favour of its protective role. We aimed to evaluate the impact of alcohol status on the hospital outcomes in patients with TBI of different mechanisms of injury (MOI).

Methods A retrospective observational analysis was conducted for all TBI patients admitted in a level 1 trauma centre between 2010 and 2014. Patients were divided into 2 groups according to the serum alcohol (negative alcohol; NATBI $v$ s positive alcohol TBI; PATBI). Patients demographics, injury severity score (ISS), head injury severity (AIS), type of TBI lesions and hospital outcomes were analysed and compared in the 2 groups in general and in relation to the MOI in terms of motor vehicle crashes (MVC),pedestrians injury, and fall from height.

Results Over the study period, 805 patients (93\% males) were admitted with TBI with a median age of 28 (1-86) years. PATBI was found in $14 \%$ of cases. Mean ISS $(\mathrm{P}=0.10)$, and hospital pneumonia $(\mathrm{p}=0.07)$, sepsis $(\mathrm{p}=0.88)$ and mortality $(\mathrm{p}=0.08)$ were fewer in PATBI in comparison to NATBI patients. In-hospital acute respiratory syndrome (ARDS) was higher in PATBI $(\mathrm{p}=0.31)$. Head AIS was greater in NATBI patients $(\mathrm{p}=0.04)$. PATBI was greater among pedestrians $(22 \%)$ 
followed by fall (13\%) and MVC (12\%). Among pedestrians, diffuse axonal injury, subarachnoid haemorrhage, skull fractures and mortality were significantly lesser in PATBI. Alcoholic pedestrians had higher proportion of polytrauma $(\mathrm{p}=0.02)$. In fall-related injury, alcoholic patients had lower proportion of intubation $(p=0.02)$ and brain oedema $(p=0.05)$. MVC-related PATBI patients had more intrahemisheric haemorrhage but less skull fracture $(p=0.02)$ and epidural haemorrhage $(0=0.03)$.

Conclusions the proportion of positive alcohol among TBI is high; however, it shows better hospital survival. Screening of alcohol among TBI gives important clues in terms of the type of TBI lesions in relation to different mechanisms of injury. Early identification of alcoholic status and its early management could be reflected in better outcome among this set of population. This analysis is limited with the lack of long-term follow-up and patients disabilities.

\section{KNOW THE SCORE: EVALUATION OF AN INTERVENTION TO REDUCE LEVELS OF DRUNKENNESS IN A UK NIGHTLIFE AREA}

Zara Quigg, Kat Ford, Nadia Butler, Katie Hardcastle, Karen Hughes. Centre for Public Health, Liverpool John Moores University, Liverpool, UK

\subsection{6/injuryprev-2016-042156.632}

Background In the UK the sale of alcohol to drunk people is illegal; however drunkenness remains common in nightlife environments. To address drunkenness in a UK nightlife area, an intervention was implemented to increase awareness of such legislation and to support bar staff compliance with the law. Methods Pre- $(\mathrm{n}=260)$ and post- $(\mathrm{n}=318)$ intervention nightlife patron surveys were implemented exploring: drinking behaviours; expectations and tolerance of drunkenness; and knowledge of the law. The post-intervention survey additionally explored public awareness and perceptions of the intervention, and potential behavioural change as a result of the intervention.

Results The majority of pre-intervention survey participants had consumed alcohol; 63\% of drinkers had preloaded. Drinkers expected units of alcohol consumed over the course of the night was 17.9 units. The majority expected their, and other people's, level of drunkenness to be high when leaving the city's nightlife. Over half believed that if someone was drunk and tried to get served alcohol in a bar in the city centre they would be served. Four in ten thought it was legal for a bar server to sell alcohol to someone who was already drunk. Post-intervention: significantly more participants knew that it is illegal for a bar server to sell alcohol to someone who is already drunk; significantly less agreed that getting drunk is socially acceptable; and significantly less reported preloading.

Conclusions The intervention presents an important step in working towards preventing the sale of alcohol to drunks and reducing associated harms. The evaluation suggests that the intervention may have increased knowledge of the laws around the service of alcohol to drunks amongst nightlife users, changed the perceived acceptability of drunkenness and decreased preloading. Despite this, post-intervention surveys illustrated that excessive alcohol consumption and drunkenness remain key features of the night-time economy.

\section{DIVERGENT TRENDS IN EMERGENCY DEPARTMENT VISITS FOR POISONINGS BY INTENT IN VÄRMLAND, SWEDEN}

Syed Moniruzzaman. Risk Management, Karlstad University, Sweden

10.1136/injuryprev-2016-042156.633

Background Poisoning is one of the key categories of adverse events that cause a large number of hospital admissions. With the recent availability of detailed injury data it is possible to provide useful information about the demographic and clinical characteristics of patients with poisonings that warrant additional analysis and is important for policy implications. The aim of this study is to review emergency department (ED) visits for poisonings reported through Injury database (IDB), Värmland, and to evaluate demographic and clinical characteristics of poisonings by intent.

Methods Using data from the IDB, Värmland, we compared poisoning trends by poisoning intent (accidental vs suicide attempt) from 2007 to 2013 . We also analysed poisoning cases by age, sex and mechanism of intoxication using distribution tabulation and logistic regression.

Results According to the IDB data, ED visits for suicide attempt by poisonings showed continuous increases from 2007 to 2013. Continuous and somewhat sharp increases were seen in suicide attempt by poisonings resulting from prescribed drugs during the period. By contrast, levelling off or steady declines in accidental poisonings were observed both in all poisoning cases and poisonings resulting from prescribed medicine. Age, sex, year and mechanism of intoxication were significantly associated with suicide attempt by poisonings among the ED visits due to poisoning cases.

Conclusions Inequalities in suicide attempt by poisonings exist between ages and sexes. Significant increasing trends of these self-inflicted poisonings, especially resulting from prescribed medicine, show in ED visits for poisonings when analysing IDB, Värmland database, which must be further compared with other Swedish IDBs to be resolved. The IDB data provide an opportunity to better understand poisoning mechanisms and associated risk factors, which is important for poisoning prevention practice and research.

\section{Arctic safety and occupational safety}

\section{Post Tue 2.2}

\section{REDUCING SLIPPING AND FALLING ACCIDENTS IN MINING AND QUARRYING INDUSTRY IN THE ARCTIC AREA BY SELCTION OF SUITABLE SAFETY FOOTWEAR}

Mikko Hirvonen. Finnish Institute of Occupational Health, Finland

10.1136/injuryprev-2016-042156.634

Background Accidents related to slips, trips and falls represent roughly one fourth of the total accidents in the mining and quarrying industry in Finland. According to a recent estimate, in Finland, an accident involving a fall costs about $6000 €$ on average. Working in the northern open pit mines exposes the workers in harsh weather conditions where pedestrian walkways are often very slippery because they are covered by ice and snow. Therefore it is essential that the footwear used by the workers has good grip in those conditions. 